\title{
EuroHYP-1: European multicenter, randomized, phase III clinical trial of therapeutic hypothermia plus best medical treatment vs. best medical treatment alone for acute ischemic stroke
}

\author{
H. Bart van der Worp'*, Malcolm R. Macleod², Philip M. W. Bath ${ }^{3}$, Jacques Demotes ${ }^{4}$, \\ Isabelle Durand-Zaleski ${ }^{5}$, Bernd Gebhardt ${ }^{6}$, Christian Gluud ${ }^{7}$, Rainer Kollmar ${ }^{8}$, \\ Derk W. Krieger ${ }^{9}$, Kennedy R. Lees ${ }^{10}$, Carlos Molina ${ }^{11}$, Joan Montaner ${ }^{12}$, Risto O. Roine ${ }^{13}$, \\ Jesper Petersson ${ }^{14}$, Dimitre Staykov ${ }^{15}$, Istvan Szabo ${ }^{16}$, Joanna M. Wardlaw ${ }^{17}$, Stefan Schwab ${ }^{15}$, \\ and on behalf of the EuroHYP-1 investigators ${ }^{\dagger}$
}

Correspondence: H. B. van der Worp*, Department of Neurology and Neurosurgery, Brain Center Rudolf Magnus, University Medical Center Utrecht, Heidelberglaan 100, 3584 CX Utrecht, The Netherlands.

E-mail: h.b.vanderworp@umcutrecht.nl

${ }^{1}$ Department of Neurology and Neurosurgery, Brain Center Rudolf Magnus, University Medical Center Utrecht, CX Utrecht, The Netherlands ${ }^{2}$ Division of Clinical Neurosciences, University of Edinburgh, Royal Infirmary of Edinburgh, Edinburgh, UK

${ }^{3}$ Stroke, Division of Clinical Neuroscience, University of Nottingham, Nottingham, UK

${ }^{4}$ Inserm, Institut de Santé Publique, Paris, France

${ }^{5}$ Department of Public Health, AP-HP, Henri Mondor-Albert Chenevier Hospitals, Creteil, France

${ }^{6}$ Center for Clinical Studies, Erlangen, Germany

${ }^{7}$ The Copenhagen Trial Unit, Centre for Clinical Intervention Research, Copenhagen University Hospital, Copenhagen, Denmark

${ }^{8}$ Clinic for Neurology and Neurogeriatrics, Darmstadt, Germany

${ }^{9}$ Department of Neurology, Stroke Center, Copenhagen University Hospital, Copenhagen, Denmark

${ }^{10}$ Institute of Cardiovascular and Medical Sciences, University of Glasgow, Glasgow, UK

${ }^{11}$ Stroke Unit, Hospital Vall d'Hebron, Barcelona, Spain

${ }^{12}$ Neurovascular Research Laboratory, Department of Neurology, Hospital Vall d'Hebron, Barcelona, Spain

${ }^{13}$ Division of Clinical Neurosciences, Turku University Hospital, Turku, Finland

${ }^{14}$ Department of Neurology, Skåne University Hospital, Malmö, Sweden

${ }^{15}$ Department of Neurology, University of Erlangen-Nuremberg, Erlangen, Germany

${ }^{16}$ European Stroke Research Network for Hypothermia, Brussels, Belgium

${ }^{17}$ Neuroimaging Sciences, University of Edinburgh, Edinburgh, UK

Received: 2 December 2013; Accepted: 31 March 2014; Published online 15 May 2014

${ }^{\dagger}$ EuroHYP-1 investigators

Executive committee: R. Kollmar, D.W. Krieger, M.R. Macleod, J. Petersson, D. Staykov, S. Schwab (chair), I. Szabo, H.B. van der Worp.

Steering Committee: J. Demotes, I. Durand-Zaleski, B. Gebhardt, C. Gluud, K.R. Lees, C. Molina, J. Montaner, R. Roine, J. Wardlaw, and all members of the Executive Committee.

Data Safety and Monitoring Committee: P.M.W. Bath (chair), G.D. Murray, P.S. Scutt (unblinded statistician), F. Sterz.

Advisory Committee: M. Brainin, U. Dirnagl, W. Hacke (chair), M. Hennerici, D. Leys, S. Meairs, B. Norrving, P.A.G. Sandercock, N. Wahlgren.

Ethics Board: B. Heinrichs, N.E. Sahlin (chair), P. Sandöe.

Trial coordination: B. Colam, A. Stilper, N. Votruba.
Rationale Cooling reduced infarct size and improved neurological outcomes in animal studies modeling ischemic stroke, and also improved outcome in randomized clinical trials in patients with hypoxic-ischemic brain injury after cardiac arrest. Cooling awake patients with ischemic stroke has been shown feasible in phase II clinical trials.

Primary aim To determine whether systemic cooling to a target body temperature between 34.0 and $35.0^{\circ} \mathrm{C}$, started within six-hours of symptom onset and maintained for $\mathbf{2 4} \mathrm{h}$, improves functional outcome at three-months in patients with acute ischemic stroke.

Design International, multicenter, phase III, randomized, open-label clinical trial with blinded outcome assessment in 1500 patients aged 18 years or older with acute ischemic stroke and a National Institutes of Health Stroke Scale score of 6 up to and including 18. In patients randomized to hypothermia, cooling to a target body temperature of $34-35^{\circ} \mathrm{C}$ will be started within six-hours after symptom onset with rapid intravenous infusion of refrigerated normal saline or a surface cooling technique and maintained for $24 \mathrm{~h}$ with a surface or endovascular technique. Patients randomized to hypothermia will receive pethidine and buspirone to prevent shivering and discomfort.

Primary outcome Score on the modified Rankin Scale at 91 days, as analyzed with ordinal logistic regression and expressed as a common odds ratio.

Discussion With 750 patients per intervention group, this trial has $90 \%$ power to detect $7 \%$ absolute improvement at the $5 \%$ significance level. The full trial protocol is available at http:// www.eurohyp1.eu. ClinicalTrials.gov Identifier: NCT01833312. Key words: acute stroke therapy, clinical trial, hypothermia, ischemic stroke, treatment

Other EuroHYP-1 consortium members involved in the conduct of this trial: G. Broessner, C. Cordonnier, A. Czlonkowska, T. Dalkara, L. Garattini, C. Hobohm, D. Jatužis, R. von Kummer, A. Marcon, A. Meisel, F. Orzi, G. Tsivgoulis. G. Vanhooren, M. Wagner, P. Winkel.

Conflicts of interest: All authors are members of the Steering Committee of EuroHYP-1, except for PMWB, who is the chair of the trial's DSMC. Unconditional support to investigator meetings and meetings of the organization EuroHYP has been provided by BARD, EMCOOLS, MTRE, and ZOLL. These companies also provide cooling equipment at discount rates for the EuroHYP-1 trial and the related trial COOLIST, of which HBvdW is the chief investigator. EuroHYP-1 is conducted independently of the funders and equipment suppliers.

DOI: $10.1111 /$ ijs.12294 


\section{Introduction and rationale}

In patients with acute ischemic stroke, early intravenous thrombolysis with alteplase, the administration of aspirin, decompressive surgery, and stroke unit care are the only treatments of proven benefit (1). Even if treatments are combined, about half of the patients remain dependent or die (2). For this reason, there is a clear need for additional, effective therapeutic strategies.

In animal studies modeling ischemic stroke, cooling to $35^{\circ} \mathrm{C}$ reduced infarct size by about one-third, and cooling to $34^{\circ} \mathrm{C}$ by around $45 \%$ (3). Several prospective observational clinical studies in stroke have shown an association between raised body temperature and poor outcome (4). Finally, cooling improved outcome in randomized trials in patients with hypoxic-ischemic brain injury after cardiac arrest (5). Next to novel reperfusion strategies, hypothermia is therefore a promising treatment for patients with acute ischemic stroke.

Systematic review and meta-analysis of therapeutic hypothermia for any indication strongly suggests an association between cooling and the risk of pneumonia and sepsis. No increase in the risk of any infection was observed when cooling was used to treat ischemic stroke, but the number of patients treated was small (6). In phase II trials, cooling awake patients with ischemic stroke has been shown feasible and safe (7-10), but whether this improves functional outcome has not yet been tested in an adequately-sized randomized clinical trial.

The primary objective of EuroHYP-1 is to determine whether systemic cooling to a target body temperature between 34.0 and $35.0^{\circ} \mathrm{C}$, started within six hours of symptom onset and maintained for $24 \mathrm{~h}$, improves functional outcome at three months in patients with acute ischemic stroke.

\section{Methods}

\section{Design}

This is an international. multicenter, randomized, parallel-group, open-label clinical superiority trial with blinded outcome assessment, registered at ClinicalTrials.gov as NCT01833312. The trial will be conducted in accordance with the ethical principles that have their origin in the Declaration of Helsinki and are consistent with International Conference on Harmonization-Good Clinical Practice and applicable regulatory requirements.

\section{Eligibility}

Patients may be enrolled in the trial if all of the inclusion criteria listed in Table 1 have been met but will be excluded from participation for any of the reasons listed in Table 2.

\section{Treatment allocation}

After enrollment, patients will be allocated to hypothermia plus best medical treatment or best medical treatment alone based on proportional minimization through a web-based allocation service. Treatment allocation will be stratified by country and will include the following minimization factors: intention to give alteplase; intended method of cooling (surface or endovascular); gender; stroke severity [National Institutes of Health Stroke Scale (NIHSS) 6-12 or 13-18]; age ( $\leq 65$ years or $>65$ years); visibility of a relevant ischemic lesion on the first brain imaging (yes or no), and time since symptom onset ( $\leq 4 \mathrm{~h}$ or $4-6 \mathrm{~h}$ ).

\section{Treatment}

In patients randomized to hypothermia, cooling will be started within six hours after onset of symptoms and within 90 mins of start of thrombolysis (or within 90 mins of hospital admission in patients who are not treated with thrombolysis) with intravenous infusion of $20 \mathrm{ml} / \mathrm{kg}$ refrigerated normal saline $\left(4^{\circ} \mathrm{C}\right)$ over 30-60 mins or a surface cooling method, depending on local practice or preference. Cooling will be maintained at $34-35^{\circ} \mathrm{C}$ for $24 \mathrm{~h}$ with a surface or endovascular technique. The choice of this technique is at the discretion of the local investigator. Thereafter, patients will be passively warmed at a rate of $0 \cdot 2 \pm 0 \cdot 1^{\circ} \mathrm{C}$ per hour until the rectal or bladder temperature reaches $36 \cdot 0^{\circ} \mathrm{C}$; the cooling device will then be disconnected.

In patients randomized to hypothermia, shivering and discomfort will be prevented and treated with intravenous pethidine ( $\max 500 \mathrm{mg} / 24 \mathrm{~h}$ ) and oral buspirone ( $\max 30 \mathrm{mg} / 24 \mathrm{~h})$. The maximum dose of pethidine is about half that administered in previous trials targeting a body temperature of $33^{\circ} \mathrm{C}$ for $24 \mathrm{~h}(8,9)$ but higher than the dose of $2.3 \mathrm{mg} / \mathrm{kg}$ used in a recent surface cooling trial targeting a body temperature of $35^{\circ} \mathrm{C}$ for $12 \mathrm{~h}(10)$. In the last trial, pethidine was combined with intravenous dexmedetomidine. Patients who have difficulties swallowing will not receive buspirone unless they have a nasogastric tube. In one animal study of focal cerebral ischemia, pethidine did not affect the effects of hypothermia (11). For the prevention of

Table 1 Inclusion criteria

Diagnosis of acute ischemic stroke.

- Age $\geq 18$ years.

- NIHSS score of 6 up to and including 18.

- GCS motor response sub-scale score $\geq 5$.

mRS score $\leq 2$ prior to onset of stroke.

Estimated body weight of 50 up to and including $120 \mathrm{~kg}$

- Possibility to start therapeutic hypothermia within six hours after onset of stroke.

- Possibility to start therapeutic hypothermia within 90 mins after start of alteplase administration in patients receiving thrombolysis.

- Possibility to start therapeutic hypothermia within 90 mins after admission to trial site in patients not receiving thrombolysis.

- Written informed consent obtained from the patient or his/her legally acceptable representative or under such other arrangements as may be legally established in participating countries. 
Table 2 Exclusion criteria

- Evidence from CT or MRI of intracranial hemorrhage or tumour or encephalitis or any diagnosis likely to cause the present symptoms other than acute ischemic stroke. Hemorrhagic transformation of the infarct is not an exclusion criterion, except when there is a parenchymal hematoma covering more than $30 \%$ of the infarcted area, with significant space-occupying effect, or when there is a bleeding remote from the infarcted area.

- Rapidly resolving stroke symptoms.

- Use of monoaminoxidase inhibitors in the 14 days prior to screening.

- Current use of medication interacting with pethidine or buspirone.

- Acute alcohol intoxication.

- Opioid addiction.

- Nursing mother or pregnant woman, as verified by a positive urine pregnancy test in females of childbearing potential.

- Known hypersensitivity to the investigational medicinal products or any of their formulation ingredients.

- Any acutely life-threatening conditions other than acute ischemic stroke.

- Known convulsive disorder, acute closed angle glaucoma, myasthenia gravis.

- $\mathrm{SPO}_{2}<94 \%$ (as measured by pulse oximetry) under nasal oxygen administration.

- Other severe respiratory disorder.

- Bradycardia (<40 bpm).

- Severe cardiac failure, defined as NYHA classification $\geq$ III.

- Myocardial infarction or angina pectoris in the three months prior to screening.

- Vasospastic disorders.

- Haematological dyscrasia.

- Known platelet count $<100000 / \mathrm{mm}^{3}$.

- Known INR > 1.7.

- Skin damage at the sites intended to be used for cooling.

- Clinical diagnosis of sepsis.

- Known severe hepatic impairment (serum ALAT and/or ASAT > 3 times ULN).

- Known renal impairment (serum creatinine $>2 \mathrm{mg} / 100 \mathrm{ml}$ ).

- Addison's disease.

- Any other condition that may interfere with, or be aggravated by, therapeutic hypothermia.

- Any condition that is thought to reduce the compliance to cooperate with the trial procedures.

- Patient who is imprisoned or is lawfully kept in an institution.

- Employee or direct relative of an employee of the CRO (if applicable), the department of the investigator, or the sponsor.

- Participation in an interventional clinical trial within the last 4 weeks, or be under the exclusion period from another trial.

Prior participation in this trial.

opioid-induced nausea and vomiting, ondansetron or granisetron is recommended.

In both intervention groups, patients will be treated according to published guidelines for the treatment of acute ischemic stroke and for secondary prevention.

\section{Outcomes}

The primary outcome is the score on the modified Rankin Scale (mRS) at $91( \pm 14)$ days, as analyzed with ordinal logistic regression and expressed as a common odds ratio. Secondary outcomes include death or dependency ( $\mathrm{mRS}>2$ ), death, and scores on the NIHSS, EQ-5D-5L, and WHODAS $2 \cdot 0$ at $91( \pm 14)$ days, and infarct size, growth, swelling, and hemorrhagic transformation on repeat brain imaging with computed tomography or magnetic resonance imaging at $48 \mathrm{~h}( \pm 24)$. Blood biomarker levels will be assessed in venous blood drawn before start of trial treatment and at 24 and $72 \mathrm{~h}$ after randomization. These biomarkers include but are not limited to - gelatinases, collagenases, stromelysines, $\mathrm{D}$-dimer, and C-reactive protein. An economic evaluation including a comparison of costs and an incremental cost-utility analysis will be performed.

To allow blinded assessment of the primary outcome and to enhance statistical power, the mRS at 91 days will be assessed by at least four independent adjudicators based on a video clip of the
mRS interview, without reference to treatment allocation, coordinated from the University of Glasgow (12).

\section{Sample size}

A total of 1474 patients is required to detect an absolute reduction in the risk of death or disability (mRS $>2$ ) of $7 \%$ (corresponding to an odds ratio of 0.74 ) with $90 \%$ power at the $5 \%$ significance level (two sided) and allowing for 3\% loss to follow-up. This target sample size is rounded to 1500 patients.

\section{Statistical analyses}

The primary and secondary outcomes will be analyzed using the intention-to-treat population. The effect of the intervention on the primary outcome will also be studied in specific sub-groups, including those used as minimization factors at the time of randomization. Additional exploratory analyses will be based on the per-protocol population. The primary efficacy variable will be analyzed with ordinal logistic regression and expressed as a common odds ratio. The analysis will be adjusted for the minimization factors mentioned above. If the assumption of the ordinal regression analysis model is not fulfilled, the groups will be compared using a nonparametric method and that result will be the primary result. The secondary outcomes and safety variables will be analyzed with logistic regression, a general linear 
univariate model, or a nonparametric test, where appropriate. A detailed statistical analysis plan will be published separately before data will be analyzed.

\section{Data Safety and Monitoring Committee (DSMC)}

An independent DSMC has been established to monitor the safety of the patients in the EuroHYP-1 trial by reviewing the available clinical data after 50,100, 200, 400, 800, and 1200 patients have been recruited and followed up until the final assessment at 91 days and ad hoc as needed. With respect to efficacy and hazard, the DSMC will conduct interim analyses on partially cleaned data after the final assessment of the first 400 and 800 patients. Details are defined in a DSMC charter.

\section{Study organization}

The University of Erlangen-Nürnberg is the trial sponsor within the European Union. To qualify for participation in the trial, investigators and their staff will undergo training prior to recruiting patients into the trial. The trial will be approved by appropriate review boards in each participating center. This trial will be monitored regularly by a qualified monitor from the sponsor or an organization commissioned by the sponsor, according to GCP guidelines and the respective standard operating procedure.

\section{Funding}

EuroHYP-1 has received funding from the European Union's Seventh Framework Program (FP7/2007-2013) under grant agreement $\mathrm{n}^{\circ} 278709$. The prefunding phase was supported by an unconditional grant from W. Hacke from his 2009 Max Jarecki and Mihara awards. HBvdW is supported by a grant from the Dutch Heart Foundation (2010T075). PMB is Stroke Association Professor of Stroke Medicine.

\section{Summary and conclusions}

One of the reasons for the recurrent failure of allegedly neuroprotective strategies to improve functional outcome in patients may be that most inhibit only a single step in the chain of events leading to cell death. By contrast, hypothermia affects a wide range of cell death mechanisms, including energy depletion, disruption of the blood-brain barrier, free radical formation, excitotoxicity, and inflammation (4). The proof of principle for therapeutic hypothermia has been provided in randomized trials in patients with postanoxic encephalopathy after cardiac arrest (5), although the validity of trials' results have been questioned (13). In addition, the optimal target temperature and the effects of delay to treatment are still uncertain $(14,15)$. Next to novel reperfusion strategies, therapeutic hypothermia may therefore represent the best chance for patients with acute ischemic stroke patients to gain major benefit in terms of functional outcome.
EuroHYP-1 will test this assumption, parallel to the American Intravascular Cooling in the Treatment of Stroke 2/3 Trial (ICTuS2/3; http://clinicaltrials.gov/show/NCT01123161).

The complete EuroHYP-1 protocol may be obtained from the study sponsor and can be accessed at the study website http:// www.eurohyp1.eu. No rights can be derived from this summarized version.

\section{References}

1 Jauch EC, Saver JL, Adams HP Jr et al. Guidelines for the early management of patients with acute ischemic stroke: a guideline for healthcare professionals from the American Heart Association/American Stroke Association. Stroke 2013; 44:870-947.

2 Wardlaw JM, Murray V, Berge E et al. Recombinant tissue plasminogen activator for acute ischaemic stroke: an updated systematic review and meta-analysis. Lancet 2012; 379:2364-72.

3 van der Worp HB, Sena ES, Donnan GA, Howells DW, Macleod MR. Hypothermia in animal models of acute ischaemic stroke: a systematic review and meta-analysis. Brain 2007; 130:3063-74.

4 van der Worp HB, Macleod MR, Kollmar R. Therapeutic hypothermia for acute ischemic stroke: ready to start large randomized trials? J Cereb Blood Flow Metab 2010; 30:1079-93.

5 Arrich J, Holzer M, Herkner H, Mullner M. Hypothermia for neuroprotection in adults after cardiopulmonary resuscitation. Cochrane Database Syst Rev 2009; (4):CD004128.

6 Geurts M, Macleod MR, Kollmar R, Kremer PH, van der Worp HB. Therapeutic hypothermia and the risk of infection: a systematic review and meta-analysis. Crit Care Med 2014; 42:231-42.

7 Krieger DW, De Georgia MA, Abou-Chebl A et al. Cooling for acute ischemic brain damage (cool aid): an open pilot study of induced hypothermia in acute ischemic stroke. Stroke 2001; 32:1847-54.

8 De Georgia MA, Krieger DW, Abou-Chebl A et al. Cooling for acute ischemic brain damage (cool aid): a feasibility trial of endovascular cooling. Neurology 2004; 63:312-7.

9 Hemmen TM, Raman R, Guluma KZ et al. Intravenous thrombolysis plus hypothermia for acute treatment of ischemic stroke (ICTuS-L): final results. Stroke 2010; 41:2265-70.

10 Piironen K, Tiainen M, Mustanoja S et al. Mild hypothermia after intravenous thrombolysis in patients with acute stroke: a randomized controlled trial. Stroke 2014; 45:486-91.

11 Sena ES, Jeffreys AL, Cox SF et al. The benefit of hypothermia in experimental ischemic stroke is not affected by pethidine. Int J Stroke 2013; 8:180-5.

12 McArthur KS, Johnson PC, Quinn TJ et al. Improving the efficiency of stroke trials: feasibility and efficacy of group adjudication of functional end points. Stroke 2013; 44:3422-8.

13 Nielsen N, Friberg H, Gluud C, Herlitz J, Wetterslev J. Hypothermia after cardiac arrest should be further evaluated - a systematic review of randomised trials with meta-analysis and trial sequential analysis. Int J Cardiol 2011; 151:333-41.

14 Nielsen N, Wetterslev J, Cronberg T et al. Targeted temperature management at 33 degrees $\mathrm{C}$ versus 36 degrees $\mathrm{C}$ after cardiac arrest. $\mathrm{N}$ Engl J Med 2013; 369:2197-206.

15 Kim F, Nichol G, Maynard C et al. Effect of prehospital induction of mild hypothermia on survival and neurological status among adults with cardiac arrest: a randomized clinical trial. JAMA 2014; 311:45-52. 